(2) Open Access Full Text Article

ORIGINALRESEARCH

\title{
Anemia Severely Reduces Health-Related Quality of Life in COPD Patients Receiving Long-Term Home Non-Invasive Ventilation
}

\author{
Maximilian Wollsching-Strobel \\ Sarah Bettina Schwarz (iD \\ Tim Mathes \\ Daniel Sebastian Majorski $\mathbb{D}$ \\ Pouya Heidari \\ Doreen Kroppen \\ Friederike Sophie Magnet (iD) \\ Wolfram Windisch (ID)
}

Cologne Merheim Hospital, Department of Pneumology, Kliniken der Stadt Köln $\mathrm{gGmbH}$, Witten/Herdecke University, Faculty of Health/School of Medicine, Cologne and Witten, North Rhine-

Westphalia, Germany
Correspondence: Wolfram Windisch Cologne Merheim Hospital, Department of Pneumology, Kliniken der Stadt Köln gGmbH, Witten/Herdecke University, Faculty of Health/School of Medicine, Ostmerheimer Str. 200, Cologne and Witten, North Rhine-Westphalia, D-5I 109, Germany

Tel +49 22I $8907 \quad 8929$

Fax +4922189078305

Email windischw@kliniken-koeln.de
Purpose: To assess the influence of anemia on health-related quality of life (HRQL) in COPD patients receiving long-term non-invasive ventilation (NIV).

Patients and Methods: In this prospective single-center cohort study, COPD patients on long-term NIV were analyzed between June 2015 and May 2020. Linear multiple regression analyses were performed using the results of the Severe Respiratory Insufficiency Questionnaire (SRI) along with the following variables: sex, age, body mass index, duration of NIV, exacerbation history ( $\leq 1$ versus $>1$ in the previous year), the updated Charlson comorbidity index, hemoglobin levels and anemia (WHO criteria).

Results: Anemia was identified in $32.8 \%(\mathrm{~N}=128)$. Anemia (mean difference $-8.4,95 \% \mathrm{CI}$ $-2.0 /-14.9$ SRI points, $P=0.011$ ) and exacerbations (mean difference $-9.9,95 \% \mathrm{CI}-4.3 /$ 15.5 SRI points, $P=0.001$ ) each had a negative impact on SRI summary scores. Exacerbations were negatively associated with six out of seven SRI subscale scores, while anemia was negatively associated with four out of seven. SRI summary scores dropped by 1.5 points for every $\mathrm{g} / \mathrm{dl}$ of hemoglobin $(P=0.08)$. No other variables had an influence on the SRI scores.

Conclusion: The present study has shown that within a cohort of COPD patients undergoing long-term NIV, one-third were identified as anemic. Furthermore, anemia, like exacerbation history, was found to have a considerable negative impact on HRQL that is specific to patients with chronic respiratory failure.

Clinical Trial Registration: German Clinical Trials Registry (DRKS00008759).

Keywords: chronic respiratory failure, exacerbations, hemoglobin, non-invasive ventilation, severe respiratory insufficiency questionnaire

\section{Introduction}

Chronic obstructive pulmonary disease (COPD) is a major cause of morbidity and mortality worldwide. ${ }^{1,2}$ In advanced stages of the disease, COPD is characterized by exacerbations and a severe reduction in health-related quality of life (HRQL). ${ }^{3}$ In addition, COPD is associated with several significant co-morbidities that also have the potential to negatively impact on HRQL. ${ }^{4-7}$ To this end, anemia is increasingly being recognized in COPD patients and is also suggested to be responsible for an observed reduction in $\mathrm{HRQL}{ }^{8,9}$

As the disease progresses further, COPD patients are at high risk of developing chronic respiratory failure. As a result, long-term oxygen treatment (LTOT) has been established to treat chronic hypoxemic respiratory failure, ${ }^{10}$ while long-term 
non-invasive ventilation (NIV) is increasingly being used to treat chronic hypercapnic respiratory failure. ${ }^{11,12}$ Both of these treatment strategies are aimed at improving longterm survival and HRQL, and are even combined in some patients. $^{13}$

In modern medicine, the measurement of HRQL has become an important means of subjectively assessing health impairments. ${ }^{14}$ Disease- or condition-specific instruments are postulated to have the highest level of sensitivity when specific treatment interventions are being assessed, or when a specific patient cohort is being investigated. ${ }^{14}$ Here, the Severe Respiratory Insufficiency Questionnaire (SRI) is a well-validated HRQL measure for COPD patients with chronic respiratory failure. ${ }^{15-17}$

Several studies have previously used this highly specific questionnaire to assess HRQL in COPD patients with chronic respiratory failure. ${ }^{13,15,16,18}$ However, the impact of anemia on HRQL in the subgroup of COPD patients who are most heavily affected by this condition - ie those with chronic hypercapnic respiratory failure - has not yet been investigated. Therefore, the present study aimed to assess the influence of those aspects of HRQL specific to chronic respiratory failure in COPD patients receiving long-term NIV, with specific emphasis on anemia. Thereby, it was hypothesized that anemia negatively affects HRQL in these patients.

\section{Methods}

The study was conducted as a single-centre observational cohort study at the Department of Pneumology, CologneMerheim Hospital, University Witten/Herdecke, Cologne. The study protocol for data acquisition was approved by the Ethics Committee for Human Studies at the University of Witten/Herdecke, Witten. Data collection was performed in accordance with the ethical standards laid down in the Declaration of Helsinki (last revision: 2013) ${ }^{19}$ and written informed consent was obtained from all subjects or their legal guardian. The cohort study from which the data were analyzed was prospectively registered at the German Clinical Trials Register (DRKS00008759). The STROBE statements for cohort studies were used as a guideline for presenting the results of this study. ${ }^{20}$

\section{Data Collection and Patient Characteristics}

Data collection was performed between June 2015 and May 2020. All patients allocated to long-term home NIV for more than one month were screened for study eligibility. Patients were only included if their chronic respiratory failure was primarily attributable to COPD. Patients primarily allocated to long-term home NIV for reasons other than COPD were excluded from the study. COPD patients with regularly scheduled follow-up visits as well as those with acute problems were included. Thus, patients status was documented as inpatient or outpatient as described in previous studies that used data from the same cohort. ${ }^{21}$

Data were collected via patient interviews and from the hospital database. The frequency of exacerbations in the year prior to study inclusion was documented and dichotomized as $\leq 1$ or $\geq 2$ exacerbation(s), according to the GOLD criteria. $^{22}$ Comorbidities were identified using the updated Charlson comorbidity index. ${ }^{23}$ Hemoglobin levels were documented from a venous blood sample taken on the same day that patients completed the SRI questionnaire.

Data on sex, age, body mass index, and duration of NIV were documented. In addition, the initial reason for NIV treatment was categorized into the following three groups: 1) patients with stable chronic hypercapnia, 2) patients with persistent hypercapnia following acute hypercapnic exacerbation and 3) patients who had undergone prolonged weaning from invasive mechanical ventilation. $^{24,25}$ Patient adherence was determined by a readout of the ventilator's built-in software. Oxygen supplement levels for long-term oxygen treatment were documented as those used by the patient at the time of study inclusion.

HRQL was assessed by the Severe Respiratory Insufficiency Questionnaire (SRI), which was specifically developed and validated for patients receiving long-term NIV and LTOT. ${ }^{15,17,26}$ Briefly, the SRI contains 49 items with seven subscales that measure different aspects of HRQL (Respiratory Complaints, Physical Functioning, Attendant Symptoms and Sleep, Social Relationships, Anxiety, Psychological Well-Being, Social Functioning). The subscales can be aggregated into one summary scale. Each scale produces a score (0-100), with lower scores indicating a poorer HRQL.

All data were entered into a standardised case report form (CRF), subsequently documented in a pseudonymous fashion, and archived electronically.

\section{Statistical Analysis}

The study was planned as a pilot study and a sample-size calculation was performed. This revealed that at least 120 patients needed to be included to ensure a reliable basis for 
statistical analysis. In addition, the number of variables in the regression was determined while taking into account that there should be at least 10 observations per variable.

The data are presented descriptively as mean \pm standard deviation (SD), if not otherwise stated. Two multiple linear regression analyses were performed with two different approaches to address the impact of anemia, as outlined in e-Figure 1. In both analyses (Analysis 1 and Analysis 2), the SRI scale scores served as the dependent variables. To adjust for confounding, sex, age, body mass index, duration of NIV, exacerbation history and the updated Charlson comorbidity index served as independent variables. In addition, directly measured hemoglobin levels were included as independent variables. The original hemoglobin values were used in Analysis 1, and the dichotomized groups were used in Analysis 2 as described as follows. Based on their hemoglobin levels, the groups were defined as "anemic" and "non-anemic" in accordance with the World Health Organization's definition of anemia for females $(\mathrm{Hg}<12 \mathrm{~g} / \mathrm{dl})$ and males $(\mathrm{Hg}$ $<13 \mathrm{~g} / \mathrm{dl}) .{ }^{27}$ Furthermore, women and men with hemoglobin levels $>15$ and $>17 \mathrm{~g} / \mathrm{dl}$, respectively, were defined as polycythemic. ${ }^{28}$ Analysis 1 addressed the general influence of reduced hemoglobin on HRQL, while Analysis 2 addressed the influence of a morbid hemoglobin deficiency - namely, anaemia - on HRQL. All confounding factors were analysed in the context of their original scales. A p-value of $<0.05$ was considered statistically significant. All analyses were performed on a complete-case basis using SPSS V.26 (IBM SPSS Statistics for Windows, 2017). Information on missing data and reasons for study exclusion are displayed in Figure 1.

\section{Results}

A total of 128 patients were enrolled for data analysis (Figure 1). NIV therapy was initiated in this patient cohort: (1) to treat stable chronic hypercapnic respiratory failure $(58 \%)$, (2) following acute NIV to treat acute respiratory failure (40\%), and (3) following prolonged weaning $(2 \%)$. Main patient characteristics, exacerbation history, hemoglobin levels, number of anemic patients, and SRI scores are displayed in Table 1. Ventilator settings were as follows: mean IPAP/EPAP settings were $23.3 \pm 4.7 / 6.0 \pm 1.3 \mathrm{cmH}_{2} \mathrm{O}$, while breathing frequency settings were $16 \pm 2$ per minute. Mean adherence to NIV was $6.3 \pm 3.5$ hours per day. Eighty percent of the patients were on LTOT in addition to long-term NIV.

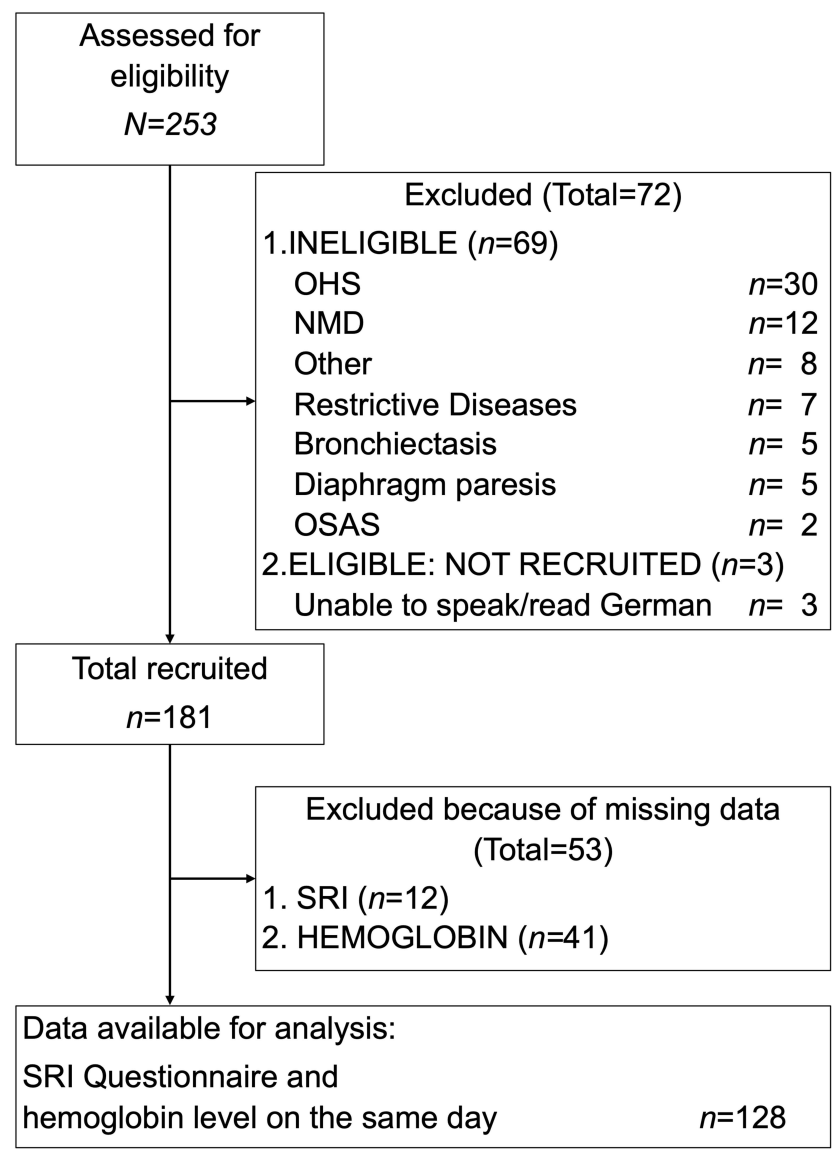

Figure I Flow diagram of subject recruitment and data availability, Abbreviations: N, number; OSAS, obstructive sleep apnea syndrome; OHS, obesity hypoventilation syndrome; NMD, neuromuscular diseases; SRI, Severe Respiratory Insufficiency Questionnaire.

Overall, $32.8 \%$ of the patients were anemic, while $4.7 \%$ had polycythemia.

According to the multiple linear regression analyses, both exacerbation history (when viewed as a confounding factor) and anemia had a significant negative impact on the SRI Summary Scale score (Table 2, e-Table 1), while reduced hemoglobin levels showed a non-significant, but clear trend towards a poorer SRI Summary Scale score ( $P=0.08$, e-Table 1). Regression coefficients for both exacerbation history and hemoglobin levels (Analysis 1) relative to the SRI Summary Scale and SRI subscale scores are shown in e-Table 2. Regression coefficients for both exacerbation history and anemia (Analysis 2) depending on the SRI Summary Scale score and the SRI subscale scores are presented in Figure 2 and e-Table 3. Finally, 95\% confidence intervals for the regression coefficient used in the multiple linear regression analysis (Analysis 2) based on the SRI scale scores are shown in Figure 3 (exacerbation history) and Figure 4 (anemia). 
Table I Patient Characteristics, Exacerbation History, Hemoglobin Levels, Number of Anemic Patients, and SRI Scores $(n=128$, if Not Otherwise Stated)

\begin{tabular}{|c|c|}
\hline & Mean \pm SD \\
\hline Age [years] & $65.6 \pm 8.1$ \\
\hline Female/male [\%] & $46.1 / 53.9$ \\
\hline Body mass index $\left[\mathrm{kg} / \mathrm{m}^{2}\right]$ & $27.4 \pm 8.3$ \\
\hline *Smoking [pack years] & $56.0 \pm 25.9$ \\
\hline Duration of NIV [years] & $1.7 \pm 1.8$ \\
\hline $\begin{array}{l}\text { Exacerbation history (preceding } 12 \text { months) }(\mathrm{N}=124) \\
\leq 1 \text { exacerbation } / \geq 2 \text { exacerbations }[\%]\end{array}$ & $56.5 / 43.5$ \\
\hline Updated Charlson Comorbidity Index ( $N=127)$ & $2.3 \pm 1.9$ \\
\hline Hemoglobin level [g/dl] & $13.1 \pm 1.9$ \\
\hline $\begin{array}{l}\text { Anemia [\%] } \\
\text { - Male [\% of all anemic patients] } \\
\text { - Female [\% of all anemic patients] }\end{array}$ & $\begin{array}{l}32.8 \\
64.3 \\
35.7\end{array}$ \\
\hline SRI summary scale & $50.3 \pm 17.5$ \\
\hline SRI subscale scores & \\
\hline - Respiratory complaints & $48.6 \pm 20.7$ \\
\hline - Physical functioning & $34.3 \pm 23.2$ \\
\hline - Attendant symptoms and sleep & $56.2 \pm 23.0$ \\
\hline - Social relationships & $66.8 \pm 21.5$ \\
\hline - Anxiety & $44.3 \pm 25.8$ \\
\hline - Psychological well-being & $53.8 \pm 20.5$ \\
\hline - Social functioning & $47.8 \pm 21.7$ \\
\hline
\end{tabular}

Note: $* 76 \%$ former smokers and $24 \%$ current smokers.

Abbreviations: SD, standard deviation; NIV, non-invasive ventilation; SRI, Severe Respiratory Insufficiency Questionnaire.

\section{Discussion}

This observational study assessed the effects of anemia, as well as several other factors, on HRQL in COPD patients receiving long-term NIV therapy. The main findings were as follows: (1) one third of all patients were anemic despite most of them being dependent on supplementary
LTOT for the treatment of hypoxemia, while the number of patients with polycythemia was negligible. In addition, there was a predominance of male patients with anemia; (2) anemia and exacerbation history were found to have the largest negative impact on HRQL in this patient cohort, while other important clinical parameters such as BMI, duration of NIV, sex, and, notably, age did not elicit negative effects. Interestingly, the number and weighting of comorbidities did not negatively impact on HRQL either; (3) exacerbation and anemia primarily affected the psychological and social domains of HRQL, but physical and functional aspects were affected to a lesser degree. The impact of both anemia and exacerbation history is suggested to be of major clinical importance. In particular, the current study is the first to establish anemia as an independent and major determinant of reduced HRQL in COPD patients with chronic respiratory failure, based on measurements using a highly precise instrument.

The current study has some important clinical implications. First, the mean difference between SRI Summary Scale scores in anemic versus non-anemic patients was more than 8 points, which is considerably higher than the previously established, minimal clinically important difference of 5 points. ${ }^{29}$ Accordingly, the mean difference in SRI Summary Scale scores in terms of exacerbation was even higher (nearly 10 points). Moreover, SRI Summary Scale scores dropped by 1.5 points for every g/dl of hemoglobin. Even though this only tended towards statistical significance, it clearly underlines the importance of reduced hemoglobin levels in COPD patients with chronic hypercapnic respiratory failure.

Previous investigations have also postulated that anemia is a negative predictor of HRQL in COPD patients. $^{8,30-32}$ However, most patients in these studies had milder forms of COPD and were not dependent on long-term NIV. In addition, the impact of anemia in some

Table 2 Multiple Linear Regression Analysis, with the SRI Summary Scale Score as the Dependent Variable $(\mathrm{N}=128)$

\begin{tabular}{|l|l|l|}
\hline Independent Variables and Confounders & Regression Coefficient 95\% Cl (Lower/Upper Limit) & $\boldsymbol{P}$-value \\
\hline Age (for each additional year) & $0.133 ;-0.235 / 0.502$ & 0.475 \\
Sex (women vs men) & $0.274 ;-5.756 / 6.304$ & 0.928 \\
BMI (for each additional index-point) & $-0.209 ;-0.581 / 0.164$ & 0.270 \\
Duration of NIV (for each additional month) & $-0.076 ;-0.213 / 0.061$ & 0.273 \\
*Exacerbation history (positive vs negative) & $-9.899 ;-15.455 /-4.344$ & 0.001 \\
Updated Charlson Comorbidity Index (for each additional index-point) & $0.562 ;-1.012 / 2.136$ & 0.481 \\
*Anemia (positive vs negative) & $-8.438 ;-14.901 /-1.975$ & 0.011 \\
\hline
\end{tabular}

Note: *Dichotomized categories as defined in the text.

Abbreviations: Cl, confidence interval; NIV, non-invasive ventilation; SRI, Severe Respiratory Insufficiency Questionnaire; BMI, body mass index. 
Regression coefficients of anemia (grey) and exacerbation history (black) in SRI scores

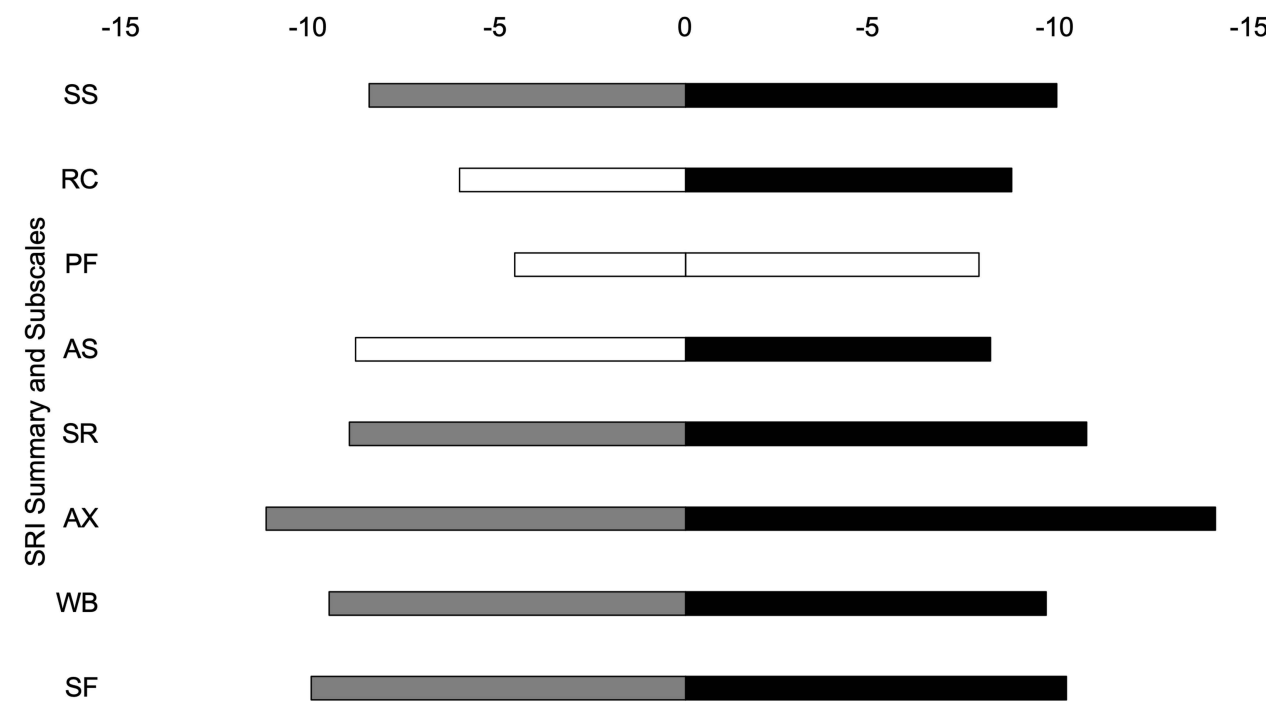

Figure 2 Regression coefficients for anemia and exacerbation history in relation to SRI scores.

Notes: Colored bars (grey for anemia, black for exacerbation history) indicate statistical significance $(P<0.05)$; white bars indicate non-significance $(P>0.05)$.

Abbreviations: SRI, Severe Respiratory Insufficiency Questionnaire; SS, Summary Scale; RC, Respiratory Complaints; PF, Physical Functioning; AS, Attendant Symptoms and Sleep; SR, Social Relationships; AX, Anxiety; WB, Psychological Well-Being; SF, Social functioning.

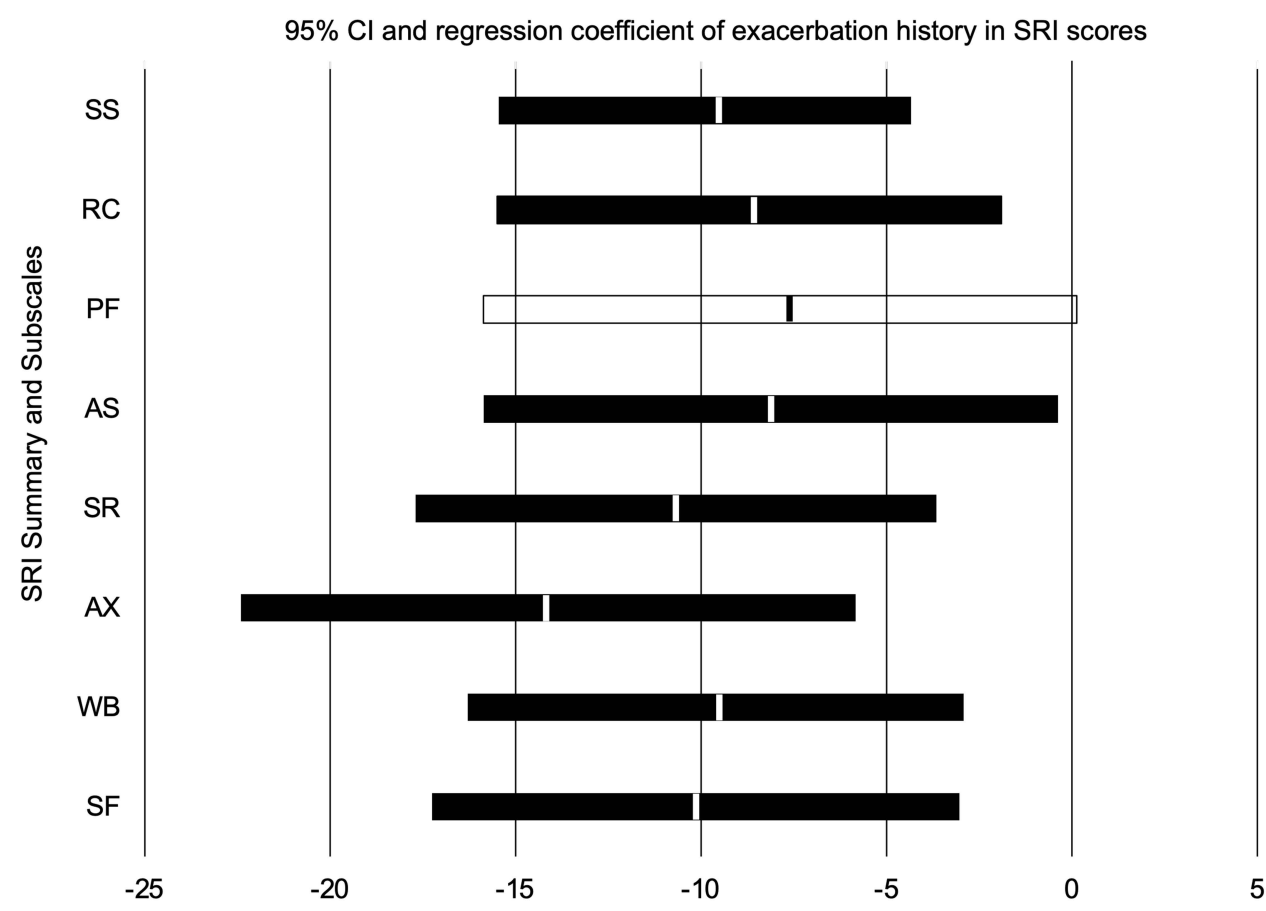

Figure $395 \%$ confidence intervals for the regression coefficient of SRI scores used in the multiple linear regression analysis for exacerbation history.

Notes: Black bars (exacerbation history) indicate statistical significance $(P<0.05)$; white bars indicate non-significance $(P>0.05)$; vertical line within each bar represents the regression coefficient.

Abbreviations: SRI, Severe Respiratory Insufficiency Questionnaire; SS, Summary Scale; RC, Respiratory Complaints; PF, Physical Functioning; AS, Attendant Symptoms and Sleep; SR, Social Relationships; AX, Anxiety; WB, Psychological Well-Being; SF, Social functioning.

of these studies was also partly attributed to comorbidities. ${ }^{8}$ Thus, the present study suggests that anemia is capable of negatively influencing HRQL, particularly in
COPD patients with severe chronic respiratory failure. Moreover, the impact of anemia on HRQL observed in the present study was highly similar to that of exacerbation 


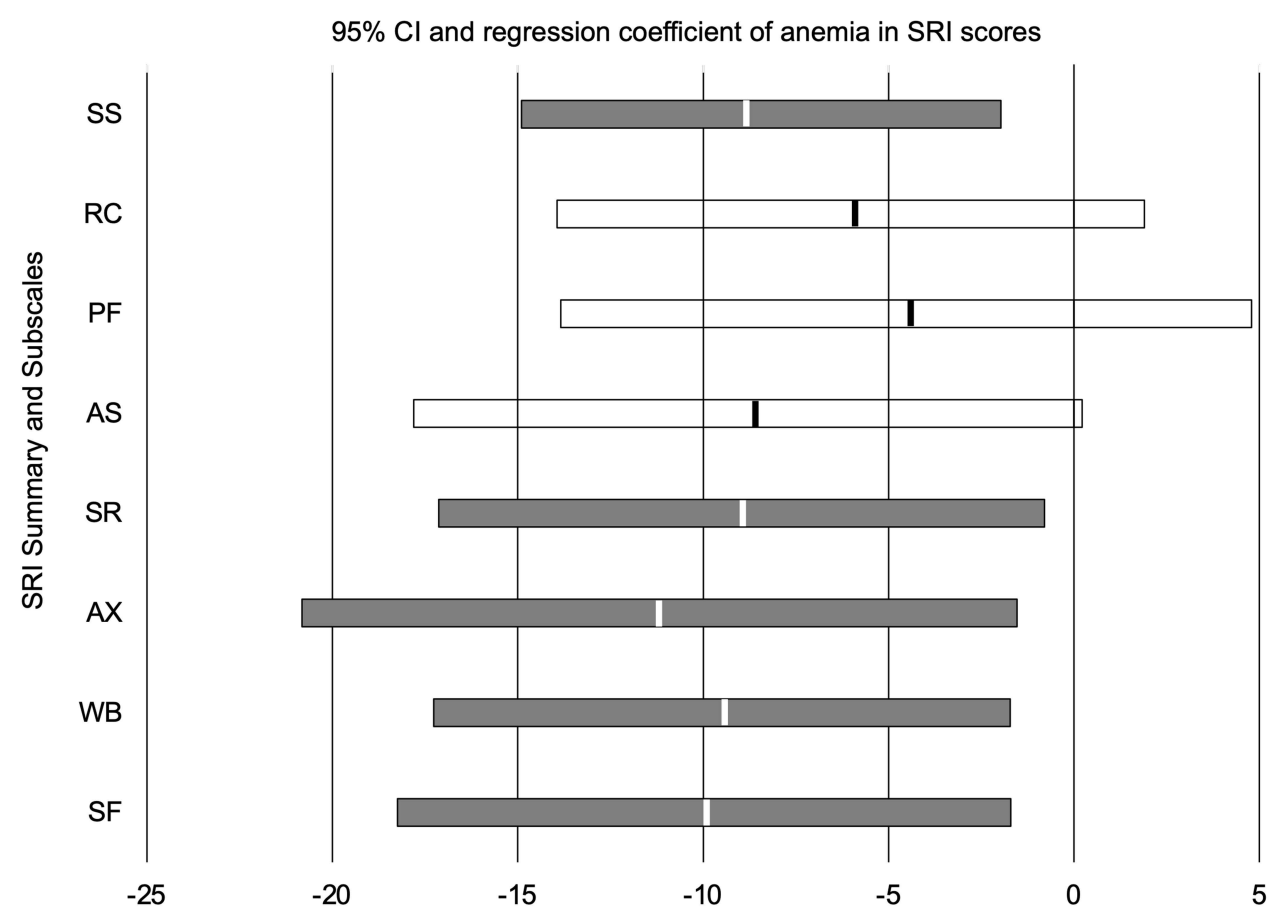

Figure $495 \%$ confidence intervals for the regression coefficient of SRI scores used in multiple linear regression analysis for anemia.

Notes: Grey bars (anemia) indicate statistical significance $(P<0.05)$; white bars indicate non-significance $(P>0.05)$; vertical line within each bar represents the regression coefficient.

Abbreviations: SRI, Severe Respiratory Insufficiency Questionnaire; SS, Summary Scale; RC, Respiratory Complaints; PF, Physical Functioning; AS, Attendant Symptoms and Sleep; SR, Social Relationships; AX, Anxiety; WB, Psychological Well-Being; SF, Social functioning.

history, which has been defined in numerous trials as a major negative factor in terms of HRQL. ${ }^{33-35}$ Accordingly, the prevention of exacerbations has been defined as a major goal in the treatment of COPD, and this is also aimed at maintaining HRQL. Therefore, the successful treatment of anemia might also serve as a means of improving HRQL in these patients. This particularly holds true in view of clinical findings in patients with cancer of different origins, in whom HRQL has been suggested to improve following successful treatment of anemia. ${ }^{36,37}$ However, whether this is also pertains to patients suffering from advanced COPD with chronic respiratory failure remains speculative, and hence needs to be elucidated in future clinical trials.

There is also an ongoing debate about whether longterm NIV should be instituted in COPD patients with chronic hypercapnic respiratory failure. Here, a recent task force of the European Respiratory Society has conditionally supported the application of long-term NIV to improve health outcome in these patients. ${ }^{38}$ Specifically, the positive effects of long-term NIV on HRQL were identified according to this analysis. In contrast, a recent meta-analysis postulated the opposite to be true, since there was no difference in HRQL in patients with versus without long-term NIV. Clearly, this discrepancy could be explained by the fact that different studies were included in the analysis, or even by the different measurement tools used to assess HRQL. ${ }^{11}$ Based on the current findings, this discrepancy might also be explained by the disparate number of anemic patients across various studies. This is supported by the observation that the prevalence of anemia is strongly heterogeneous (range: $7.5-34 \%$ ), depending on the study cohort. ${ }^{31}$ Based on this, future studies investigating long-term NIV in COPD patients should also address the role of anemia in determining HRQL and clinical outcome.

Another interesting observation refers to the updated Charlson Comorbidity Index. ${ }^{23}$ Of note, the primary aim of this update was to predict the probability of mortality within 12 months, however, in the present study, this particular index did not have a negative impact on HRQL, without even a tendency towards significance when used as a confounder. This clearly emphasizes the notion that factors influencing HRQL are not comparable with factors that influence mortality and should therefore be considered independently from one another. This 
reemphasizes the importance of reduced HQRL associated with anemia in advanced COPD patients to determine the proper therapeutic goal.

The present study has some limitations. Firstly, because both inpatients and outpatients were enrolled, the current cohort could be deemed somewhat heterogeneous. However, HRQL assessment with the SRI was based on the circumstances that occurred in the two weeks prior to enrolment, when all patients were at home. In addition, the aim of the study was to establish a real-life clinical setting and enroll a large number of COPD patients with severe chronic respiratory insufficiency. Moreover, it was ensured that only COPD patients were included, while those with other primary causes of chronic respiratory failure were excluded. The data do not allow general speculation on the etiology of anemia in this cohort. However, since only patients allocated to long-term home NIV for more than one month were included we consider possible short-term effects of repeated blood draws during prolonged weaning to be minimal. Therefore, the current cohort is suggested to reflect a real-life clinical setting. Another issue is that the level of autonomy impairment was not considered to be a potential predictor of HRQL in the present trial; ${ }^{21}$ this therefore needs to be addressed in future trials.

\section{Conclusions}

In conclusion, the present study has shown that within a cohort of COPD patients undergoing long-term NIV, one third were identified as anemic. Furthermore, like exacerbation history, anemia plays a highly important role in reducing HRQL in patients with chronic respiratory failure, whereas other important clinical parameters and potential confounding factors such as body mass index, duration of NIV, sex, age and comorbidities had no impact on HRQL in these patients.

\section{Abbreviations}

AS, attendant symptoms and sleep; AX, Anxiety; BMI, body mass index; $\mathrm{CI}$, confidence interval; $\mathrm{CRF}$, case report form; COPD, chronic obstructive pulmonary disease; EPAP, expiratory positive airway pressure; GOLD, global initiative for chronic obstructive lung disease; $\mathrm{Hg}$, hemoglobin; HRQL, health-related quality of life; IPAP, inspiratory positive airway pressure; LTOT, long-term oxygen treatment; NIV, non-invasive ventilation; NMD, neuromuscular diseases; OHS, obesity hypoventilation syndrome; OSAS, obstructive sleep apnea syndrome; PF, physical functioning; RC, respiratory complaints; SD, standard deviation; SF, social functioning; SR, social relationships; SRI, severe respiratory insufficiency questionnaire; SS, summary scale; uCCI, updated Charlson comborbidity index; WB, psychological well-being; WHO, World Health Organization.

\section{Data Sharing Statement}

The authors intend to share individual deidentified participant data with any other unfunded or funded researchrelated purpose under appropriate circumstances. Please contact the corresponding author for more information.

\section{Ethics Approval, Clinical Trial Registry and Informed Consent}

The study protocol was approved by the ethics committee at Witten/Herdecke University. The study was performed in accordance with the ethical standards laid down in the Declaration of Helsinki and was registered at the German Clinical Trials Registry (DRKS00008759). All patients provided written informed consent.

\section{Acknowledgments}

Drafts from this work were presented at the local online DGP Congress in June 2021.

\section{Author Contributions}

All authors made substantial contributions to conception and design, acquisition of data, or analysis and interpretation of data; took part in drafting the article or revising it critically for important intellectual content; agreed to submit to the current journal; gave final approval of the version to be published; and agree to be accountable for all aspects of the work.

\section{Funding}

The authors did not receive special funding for this study.

\section{Disclosure}

MWS, TM, PH, DK: no conflict of interests. SBS received travel grants from companies dealing with mechanical ventilation products. DSM reports open research grant from Philips Respironics/USA. FSM and WW received speaking fees from companies dealing with mechanical ventilation products. The study group (MWS, DSM, SBS, FSM and WW) received open research grants from Weinmann/ Germany, Vivisol/Germany, Löwenstein Medical/Germany, VitalAire/Germany, and Philips Respironics/USA. The authors report no other conflicts of interest in this work. 


\section{References}

1. Mathers CD, Loncar D, Samet J. Projections of global mortality and burden of disease from 2002 to 2030. PLoS Med. 2006;3(11):e442. doi:10.1371/journal.pmed.0030442

2. Mannino DM. COPD: epidemiology, prevalence, morbidity and mortality, and disease heterogeneity. Chest. 2002;121(5):121S-126S.

3. Seemungal TA, Donaldson GC, Paul EA, Bestall JC, Jeffries DJ, Wedzicha JA. Effect of exacerbation on quality of life in patients with chronic obstructive pulmonary disease. Am J Respir Crit Care Med. 1998;157(5):1418-1422.

4. Sundh J, Ställberg B, Lisspers K, Montgomery SM, Janson C. Comorbidity, body mass index and quality of life in COPD using the clinical COPD questionnaire. COPD. 2011;8(3):173-181.

5. Ketelaars C, Schlösser M, Mostert R, Abu-Saad HH, Halfens R, Wouters E. Determinants of health-related quality of life in patients with chronic obstructive pulmonary disease. Thorax. 1996;51 (1):39-43.

6. Lee H, Jhun BW, Cho J, et al. Different impacts of respiratory symptoms and comorbidities on COPD-specific health-related quality of life by COPD severity. Int $J$ Chron Obstruct Pulmon Dis. 2017; 12:3301.

7. Esteban C, Arostegui I, Aramburu A, et al. Predictive factors over time of health-related quality of life in COPD patients. Respir Res. 2020;21:1-11.

8. Krishnan G, Grant BJ, Muti PC, et al. Association between anemia and quality of life in a population sample of individuals with chronic obstructive pulmonary disease. BMC Pulm Med. 2006;6(1):1-9.

9. Similowski T, Agusti A, MacNee W, Schönhofer B. The potential impact of anaemia of chronic disease in COPD. Euro Respir J. 2006;27(2):390-396.

10. Cranston JM, Crockett A, Moss J, Alpers JH. Domiciliary oxygen for chronic obstructive pulmonary disease. Cochrane Database Syst Rev. 2005;4:CD001744.

11. Wilson ME, Dobler CC, Morrow AS, et al. Association of home noninvasive positive pressure ventilation with clinical outcomes in chronic obstructive pulmonary disease: a systematic review and meta-analysis. JAMA. 2020;323(5):455-465.

12. Kohnlein T, Windisch W, Kohler D, et al. Non-invasive positive pressure ventilation for the treatment of severe stable chronic obstructive pulmonary disease: a prospective, multicentre, randomised, controlled clinical trial. Lancet Respir Med. 2014;2(9):698-705.

13. Murphy PB, Rehal S, Arbane G, et al. Effect of home noninvasive ventilation with oxygen therapy vs oxygen therapy alone on hospital readmission or death after an acute COPD exacerbation: a randomized clinical trial. JAMA. 2017;317(21):2177-2186.

14. Testa MA, Simonson DC. Assessment of quality-of-life outcomes. N Engl J Med. 1996;334(13):835-840.

15. Windisch W, Freidel K, Schucher B, et al. The Severe Respiratory Insufficiency (SRI) Questionnaire A specific measure of health-related quality of life in patients receiving home mechanical ventilation. J Clin Epidemiol. 2003;56(8):752-759.

16. Windisch W. Impact of home mechanical ventilation on health-related quality of life. Eur Respir J. 2008;32(5):1328-1336.

17. Walterspacher S, July J, Kohlhäufl M, Rzehak P, Windisch W. The Severe Respiratory Insufficiency Questionnaire for subjects with COPD with long-term Oxygen therapy. Respir Care. 2016;61 (9):1186-1191.

18. Köhnlein T, Windisch W, Köhler D, et al. Non-invasive positive pressure ventilation for the treatment of severe stable chronic obstructive pulmonary disease: a prospective, multicentre, randomised, controlled clinical trial. Lancet Respir Med. 2014;2 (9):698-705.
19. Association WM. Declaration of Helsinki-Ethical principles for medical research involving human subjects official version 2013. JAMA. 2013;310:20.

20. von Elm E, Altman DG, Egger M, Pocock SJ, Gøtzsche PC, Vandenbroucke JP. The Strengthening the Reporting of Observational Studies in Epidemiology (STROBE) statement: guidelines for reporting observational studies. Lancet. 2007;370(9596):1453-1457.

21. Schwarz SB, Mathes T, Majorski DS, et al. Living conditions and autonomy levels in COPD patients receiving non-invasive ventilation: impact on health related quality of life. BMC pulmonary medicine. 2021;21(1):1-8.

22. GOLD. Global Initiative for Chronic Obstructive Lung Disease Report. Fontana, WI; 2021. Available from: www.goldcopd.org. Accessed October 16, 2021.

23. Quan $\mathrm{H}, \mathrm{Li} \mathrm{B}$, Couris $\mathrm{CM}$, et al. Updating and validating the Charlson comorbidity index and score for risk adjustment in hospital discharge abstracts using data from 6 countries. Am J Epidemiol. 2011;173(6):676-682.

24. Windisch W, Geiseler J, Simon K, Walterspacher S, Dreher M; on behalf of the Guideline C. German national guideline for treating chronic respiratory failure with invasive and non-invasive ventilation: revised edition 2017 - Part 1. Respiration. 2018;96(1):66-97.

25. Windisch W, Geiseler J, Simon K, Walterspacher S, Dreher M; on behalf of the Guideline $\mathrm{C}$. German national guideline for treating chronic respiratory failure with invasive and non-invasive ventilation - Revised Edition 2017: Part 2. Respiration. 2018;96(2):171-203.

26. Windisch W. Quality of life in home mechanical ventilation study group. Impact of home mechanical ventilation on health-related quality of life. Eur Respir J. 2008 32(5):1328-36.

27. WHO. Haemoglobin Concentrations for the Diagnosis of Anaemia and Assessment of Severity. Geneva: Vitamin and Mineral Nutrition Information System. (WHO/NMH/NHD/MNM/11.1); 2011. Available from: http://www.who.int/vmnis/indicators/haemoglobin.pdf. Accessed May 23, 2021.

28. Cote C, Zilberberg M, Mody SH, Celli B. The prevalence of polycythemia in a chronic obstructive pulmonary disease (COPD) cohort. Chest. 2005;128(4):264S.

29. Raveling T, Kort J, Bladder G, Windisch W, Wijkstra PJ, Duiverman ML. The minimal clinically important difference of the Severe Respiratory Insufficiency questionnaire in severe COPD. Eur Respir J. 2020;56:6.

30. Xiong W, Xu M, Pudasaini B, Guo X, Liu J. The influence of anemia on one-year exacerbation rate of patients with COPD-PH. BMC Pulm Med. 2018;18(1):1-8.

31. Yohannes AM, Ershler WB. Anemia in COPD: a systematic review of the prevalence, quality of life, and mortality. Respir Care. 2011;56 (5):644-652.

32. Ferrari M, Manea L, Anton K, et al. Anemia and hemoglobin serum levels are associated with exercise capacity and quality of life in chronic obstructive pulmonary disease. BMC Pulm Med. 2015;15(1):1-8.

33. Miravitlles M, Ferrer M, Pont A, et al. Effect of exacerbations on quality of life in patients with chronic obstructive pulmonary disease: a 2 year follow up study. Thorax. 2004;59(5):387-395.

34. Niewoehner DE. The impact of severe exacerbations on quality of life and the clinical course of chronic obstructive pulmonary disease. Am J Med. 2006;119(10):38-45.

35. Esteban C, Quintana JM, Moraza J, et al. Impact of hospitalisations for exacerbations of COPD on health-related quality of life. Respir Med. 2009;103(8):1201-1208.

36. Quirt I, Robeson C, Lau C, et al. Epoetin alfa therapy increases hemoglobin levels and improves quality of life in patients with cancer-related anemia who are not receiving chemotherapy and patients with anemia who are receiving chemotherapy. $J$ Clin Oncol. 2001;19(21):4126-4134. 
37. Jones M, Schenkel B, Just J, Fallowfield L. Epoetin alfa improves quality of life in patients with cancer: results of a metaanalysis. Cancer. 2004;101(8):1720-1732.
38. Ergan B, Oczkowski S, Rochwerg B, et al. European Respiratory Society guidelines on long-term home non-invasive ventilation for management of COPD. Eur Respir J. 2019;54:3.

\section{Publish your work in this journal}

The International Journal of COPD is an international, peer-reviewed journal of therapeutics and pharmacology focusing on concise rapid reporting of clinical studies and reviews in COPD. Special focus is given to the pathophysiological processes underlying the disease, intervention programs, patient focused education, and self management protocols. This journal is indexed on PubMed Central, MedLine and CAS. The manuscript management system is completely online and includes a very quick and fair peer-review system, which is all easy to use. Visit http://www.dovepress.com/testimonials.php to read real quotes from published authors. 